\title{
Acquisition of Gradual Knowledge
}

\author{
Rose DIENG, Olivier CORBY, Stéphane LAPALUT \\ ACACIA Project, INRIA \\ 2004 Route des Lucioles, BP 93 \\ 06902 SOPHIA-ANTIPOLIS CEDEX, FRANCE \\ \{dieng,corby,lapalut\}@sophia.inria.fr
}

\begin{abstract}
Topoi are gradual inference rules, often used by experts in several problem classes: they can be exploited at various phases of a knowledge-based system life cycle. They can be studied at the two levels distinguished by Newell: the knowledge level and the symbol level. Some knowledge elicitation techniques such as rating grids and some knowledge acquisition methods such as KADS and KOD can be exploited in order to facilitate the acquisition of topoi. At the symbol level, different representations and implementations of topoi can be proposed and topoi can be formalized through several qualitative physics formalisms.
\end{abstract}

\section{Introduction}

The notion of topoi ${ }^{1}$ stems from Anscombre and Ducrot's argumentation theory [2]. A topos is a gradual inference rule such as "The more $P$, the more $Q$ ", "The less $P$, the less $Q$ ", "The more $P$, the less $Q$ " or "The less $P$, the more $Q$ ", where $P$ and $Q$ are properties. The first two topoi are called positive topoi and the last two negative topoi. Several researchers studied the interest of such gradual knowledge for argumentation in linguistics [12, 41] and for artificial intelligence: in particular, in the framework of knowledge-based systems (KBS), some researchers studied thoroughly the use of topoi for knowledge acquisition $[23,27]$, reasoning $[40,9]$, explanations $[20,41,21]$, specification and validation of a KBS [18], design applications [4].

Let us give a brief description of such research. In $[12,41]$, the authors propose a topoi-based theory of argumentation in natural language. Topoi are seen as gradual rules that are applied to some elements of the signification of a phrase, and help to determine the argumentative orientation of the concerned utterance. A speaker chooses the topoi he supposes shared by his interlocutors. Topoi play an important role for representing expertise. In [41], the author distinguishes descriptive topoi and heuristic topoi and exploits the notion of topoi for explanations of an expert system. In [40], the author relies on the possibility theory and on fuzzy sets in order to reason on gradual rules. The dynamic gradual behaviour of a KBS is represented through a topoi base in [18]. The notion of conditional topoi is introduced in order to avoid conflicts between apparently contradictory topoi. The topoi base is exploited for specification and validation

1 The term "topoi" was chosen as a tribute to Aristotle's topoi. 
of an expert system. In [9], fuzzy implication is used in order to exploit gradual rules expressing topoi. In [14], the authors study the link between the three main theories of qualitative physics and the topoi-based model of the linguistic theory of argumentation. They exploit such links for generating causal explanations. In [4], the author notices that, during evaluation of solutions, the designers rely on knowledge elements called evaluative referents ; when such referents are gradual, they can be connected through topoi. In [27], the author studies acquisition of gradual knowledge for a system of aid to decision in highway maintenance.

All such research attests the interest of topoi for artificial intelligence. In our own past research concerning KBS, we studied topoi in the framework of two research topics:

- for explanations: in order to allow a KBS to provide qualitative explanations, we studied the automatic generation of topoi from an expert system rule base and we focused on the problems of consistency raised by such an automatic generation [20]. We considered topoi as relations that may label the KBS reasoning tree, for explanatory purposes [21]. Then, we exploited the obtained topoi in order to supply qualitative explanations on the KBS reasoning.

- for knowledge acquisition and validation: the past experiments of our team in design applications had revealed the frequent use of topoi by designers during their discussions with the knowledge engineer. So, we proposed a knowledge acquisition tool, aimed at design applications and based on topoi $[23,25,22]$. The validation of the acquired knowledge was enabled by a qualitative simulation relying on topoi propagation. In [26], this tool was extended with the notion of conditional topoi.

This previous research convinced us of the interest of topoi, and not only for design applications. We are now interested in studying thoroughly topoi as a specific type of knowledge to be acquired. This choice of topoi is, of course, motivated by our past research. In this article, we try to answer the following questions: how to help the knowledge engineer to recognize the need to build a knowledge-based system having gradual knowledge? And how to help him to elicit the needed topoi? We aim at generalizing later our approach for the acquisition of other specific types of knowledge.

This paper is structured as follows. First, we distinguish two viewpoints on topoi, relying on Newell's research: the knowledge level and the symbol level. Then, we show how some knowledge elicitation techniques and some well known knowledge acquisition methods allow the acquisition of topoi in a natural way. Last, at the symbol level, we evoke briefly different possible representations of topoi and the link between qualitative physics and topoi, as our privileged application domain is engineering.

\section{Two Viewpoints on Topoi}

In [38], Newell introduces the notion of knowledge level that Steels interprets as follows [45]: "the knowledge level describes intelligent systems in terms of the 
knowledge they contain rather than the structures they have that implement that knowledge... The knowledge level is a level of abstraction for studying knowledge and expertise. It focus on knowledge and knowledge use rather than representations and programming constructs."

Studying topoi at the knowledge level allows to guide their acquisition independently of the way they will be later represented and implemented.

Here are some problems that can be studied at the knowledge level:

- the link between topoi and causality: topoi can correspond to causal relations but they can also express simple correlations without any known physical or mathematical justification.

- the temporal aspect underlying topoi: if the topos "The more $P$, the more $Q$ " means that the truth of property $\mathrm{P}$ must be established temporally before the truth of property $\mathrm{Q}$, this topos is not equivalent to the topos "The more $Q$, the more $P$ ".

- the link between topoi and monotonic functions: if $\mathrm{X}$ and $\mathrm{Y}$ are variables, the topos "The more $X$ is $A$, the more $Y$ is $B$ " can mean that $Y$ is calculated using an increasing monotonic function of $\mathrm{X}$. But it can also express a simple correlation, without causality.

When the topos "The more $X$ is $A$, the more $Y$ is $B$ " means that $\mathrm{Y}$ is calculated by an increasing monotonic function of $\mathrm{X}$, we can then consider that the topos is equivalent to "The less $X$ is $A$, the less $Y$ is $B$ " and that "The more $X$ is $A$, the less $Y$ is $B$ " is equivalent to "The less $X$ is $A$, the more $Y$ is $B$ ". However, if the topos "The more $X$ is $A$, the more $Y$ is $B$ " does not express a monotonic function but rather corresponds to a non causal correlation, it is not equivalent to "The less $X$ is $A$, the less $Y$ is $B$ ". For example, each motorist can notice that "The higher the oil price, the higher the gasoline price" since a rise of oil price is always followed by a rise of the consumer's gasoline price! However, it can't be asserted that "The lower the oil price, the lower the gasoline price" as, in living motorist memory, the fall of petrol price is seldom followed by a fall of gasoline price !

- the meaning of conditional topoi: sometimes, the restriction of a topos validity domain is needed. If the variable $\mathrm{Y}$ is calculated by a non monotonic function of the variable $X$, it can be expressed by several conditional topoi.

- the meaning of $n$-ary topoi: the n-ary topos "The more $P_{1}$ and $P_{2}$ and ... $P_{n-1}$, the more $Q$ " could be transformed into $\mathrm{n}-1$ independent binary topoi "The more $P_{i}$, the more $Q$ " but information would be lost: in fact, the $\mathrm{n}$ 1 topoi must be interdependent. So, the notion of relations linking several topoi help express such an interdependence.

In this article, we just cite such problems without studying them thoroughly. Topoi need a deeper analysis at the knowledge level, so as to establish rigorously their semantics, but such a study goes beyond the framework of this article. 


\section{Acquisition of Topoi}

In [17], Clancey considers knowledge engineering as a "methodology to model processes qualitatively, in the form of relational networks describing causal, temporal and spatial relations". Due to the qualitative and causal aspects underlying them, topoi should play an important role during knowledge engineering, and specially during knowledge acquisition process.

Let us describe previous research that focused on acquisition of gradual knowledge. In $[23,25,22]$, we presented 3DKAT, a knowledge acquisition tool, aimed at design applications and based on the propagation of topoi corresponding to influence relations between the parameters of the system to be designed. We distinguished several types of topoi according to the nature of the so-linked variables (numerical or discrete). 3DKAT allowed to perform two kinds of reasonings based on topoi propagation : "what-if" reasoning in order to answer the question "What will happen if I perform this modification on this variable?" and "how-to" reasoning in order to answer the question "How to obtain this modification on this variable?".

In [9], the authors study the acquisition of gradual rules. In [27], examples of gradual knowledge for highway maintenance are presented. The author gives suggestions in order to allow an "intuitive" graphical capture of the gradual inference rules by the expert. In [4], the author studies the design task and notices that when designers describe the topic relations between the evaluative referents allowing them to evaluate their solutions, they sometimes state explicitly topoi in the classic form "The more ... the more", and other times they use verbs or adverbs expressing a graduality. The author also presents several examples of design applications.

\subsection{Knowledge Elicitation Techniques}

Among the classical knowledge elicitation techniques, which ones allow "naturally" the acquisition of topoi ? Direct techniques such as interviews, introspection, observations, verbal protocol analysis, questionnaire should allow to detect rather easily topoi when the expert is aware of using topoi in his description of a system, or in his reasoning to solve a problem. However, when the expert does not easily access to the topoi implicit in his reasoning, it seems more judicious to use indirect techniques as they can reveal implicit relations between entities, relations that the expert could not have indicated directly by himself: the technique of rating grids is an example of such indirect techniques.

After giving concrete examples of topoi elicited thanks to actual interviews of experts, we will study thoroughly the exploitation of rating grids for topoi elicitation, as this technique is frequently used in knowledge acquisition tools such as ETS [5, 6], KSS0 [43] or AQUINAS [7, 8].

Examples of Topoi Obtained through Interviews. In [4], interviews of designers produced several examples of topoi expressed by such designers, either 
directly or indirectly. Indeed, concerning the expressions used by the experts, the author notices that, if, in some cases, the experts use the classic form "The more ... the more", and in other cases, they can modulate the meaning of topoi through modalities such as "can", "may", "must"...

The author cites the following examples:

"If there are a lot of risks, there risk to be an extra cost."

(which is equivalent to modulate the topos "The higher the risks, the higher the cost.".)

"If the structures must be given very quickly, there may be extra costs."

(which is equivalent to modulate the topos "The lower the delay, the higher the cost.").

Here are other examples cited by the same author, examples expressing topoi with or without modulation:

"The more precise the requirements, the more new technologies must be used." "If the expert has a lot of experience, there are few risks."

"When the solution can be tested, it is all the more reliable."

"When the geometry increases, the mass increases and the frequence decreases."

Likewise, in [27], the author presents several examples of topoi appearing during interviews with the expert for the construction of a system for aid to decision in highway maintenance:

"The more there is water infiltration in the roadway body, the worse the foundation risks to be."

"The more the profiles are uneven, the worse the foundation risks to be."

"The higher the speed of the vehicles, the more important the measure of the importance relatively to the roadway comfort."

"The less homogeneous the roadway, the less comfortable it is."

"The older the roadway, the worse the foundation risks to be."

"If there is a punctual undressing and if the roadway is between five and fifteen years old, then the cause "too old coating" is all the more certain since the roadway is older.".

The author notices the ambiguity of the semantics of such rules as "The more $X$ is $A$, the more $Y$ risks to be $B$ ".

Those few examples illustrate the various forms under which the topoi can appear during the expert's interviews.

As noticed in [4], the presence, in the expert's discourse, of some verbs, adverbs or expressions that can express implicitly a graduality ("all the more so since", "increase", "decrease", "the more", "the higher", "the less", "the lower"...) can prompt the knowledge engineer to try to detect a topos, and ask the expert to validate it.

Remark: Of course, a more rigorous linguistic analysis of the expert's discourse is needed, in order to allow an actual elicitation of topoi directly from a text. Research on the role of topoi in natural language [41] should be a step towards such an analysis. 


\section{The Technique of Rating Grids.}

Overview. The rating grids allow to reveal relations between concepts or solutions, relations that the expert would hardly make explicit by himself. So this technique should be helpful to acquire gradual relations between some concepts or some solutions.

The technique of rating grids is inspired of Kelly's theory of the personal constructs [33]. However the description presented below does not correspond to the pure repertory grids, as described in Kelly's book, but rather to the pragmatic interpretation adopted by knowledge acquisition tools such as ETS $[5,6]$.

The knowledge engineer asks to the expert the main conclusion items (they generally correspond to possible solutions of the problem to be solved). Then by taking triads of those items, he asks a trait or characteristics distinguishing any two of the three elements from the other. A trait and its opposite constitute a construct. Each item is rated relatively to each construct. Different grid analysis techniques using distance-based measures between the columns (resp. the rows) of the grids help to compare the items (resp. the constructs), so as to find similarities and differences among them. For example, an item dependency tree and a characteristic dependency tree can be calculated. The results are then interpreted and validated by the expert. This technique allows to reveal similarities and differences between different items and between different characteristics: the dependency trees reveal which items or which traits are close and which ones are distant.

Topoi Elicitation. By examining the characteristic dependency tree, the knowledge engineer can then ask the expert if there exists a gradual relation between two close traits (probably, a positive topos) or between two distant traits (probably, a negative topos). By this way, the knowledge engineer can help the expert to make explicit topoi such as "The more an item has the characteristics Char 1 , the more this item has the characteristics Char ${ }_{2}$ " which are particular topoi since they concern characteristics of a same entity.

Even though the knowledge engineer has not yet built the characteristic dependency tree, the knowledge engineer can look for topoi directly in the grids indicating the rates of the items relatively to the constructs. Such rates could easily be exploited using an algorithm allowing to reveal potential topoi.

How to detect topoi concerning characteristics of different entities? If the columns of the rating grid correspond to the different possible solutions, the characteristics allowing to discriminate such solutions can concern different concepts playing a significant role for the solutions. In this case, the characteristic dependency tree will concern traits of different concepts and will reveal topoi such as "The more Concept ${ }_{1}$ has the characteristics Char ${ }_{1}$, the more Concept ${ }_{2}$ has the characteristics Char ${ }_{2}$ ". 


\section{Example.}

Car-1 Car-2 Car-3 Car-4 Car-5 Car-6 Car-7 Car-8

\begin{tabular}{|c|c|c|c|c|c|c|c|c|}
\hline Easy to handle/Not easy to ha & & 3 & 5 & 2 & 5 & 3 & 3 & \\
\hline Sportive/Not sportive & 2 & 2 & 2 & 1 & 5 & 2 & 3 & \\
\hline Safe/Light & 4 & 5 & 3 & 2 & 4 & 5 & 4 & \\
\hline Habitable/Not very habitable & 5 & 4 & 3 & 2 & 1 & 5 & 5 & \\
\hline Comfortable/Uncomfortable & 5 & 5 & 4 & 1 & 3 & 5 & 4 & \\
\hline Silent/Noisy & 4 & 4 & 5 & 2 & 2 & 4 & 4 & \\
\hline Responsive/Heavy & 2 & 2 & 3 & 1 & 5 & 2 & 4 & \\
\hline Attractive/Unattractive & 4 & 2 & 3 & 3 & 4 & 2 & 4 & \\
\hline Cheap/Expensive & 1 & 1 & 4 & 4 & 2 & 1 & 1 & \\
\hline
\end{tabular}

Fig. 1. Example: a rating grid upon 8 cars (extracted from [46])

In the example shown figure 1 , an algorithm calculating the direction of the variations of the rates on each row (i.e. for each construct) would incite the knowledge engineer to ask the expert whether there exists a positive topos between the sportivity and the responsiveness of the car, or between its sportivity and the fact it is attractive, or between its responsiveness and the fact it is attractive: such positive topoi can be suspected since the rates along the two compared rows vary in the same direction, even though sometimes, when a rate is modified on one row, it may remain constant on the other row. On the contrary, the knowledge engineer would be prompted to look for a possible negative topos between the safety and the cheapness of the car, or between the cheapness and the comfort of the car: indeed, the rates vary in opposite directions, along the two compared rows.

In the same example, if, before looking for topoi, the knowledge engineer analyzes the characteristic dependency tree shown in figure 2 , he may be incited to inquire about a possible positive topos between the sportivity and the responsiveness of the car ( $\mathrm{cf}$ the distance between both characteristics is only 2 ), or between the car safety and cheapness ( $\mathrm{cf}$ the distance is 3 ), or even between cheapness and comfort ( $\mathrm{cf}$ the distance is 4 ). He can try to find whether a. negative topos exists between sportivity and silence, or between responsiveness and silence, or between sportivity and habitability or between responsiveness and habitability (as all couples of compared characteristics are very distant, as indicated by the characteristic dependency tree).

In conclusion, the rating grids may help to reveal topoi the expert would not have been aware of, initially. 


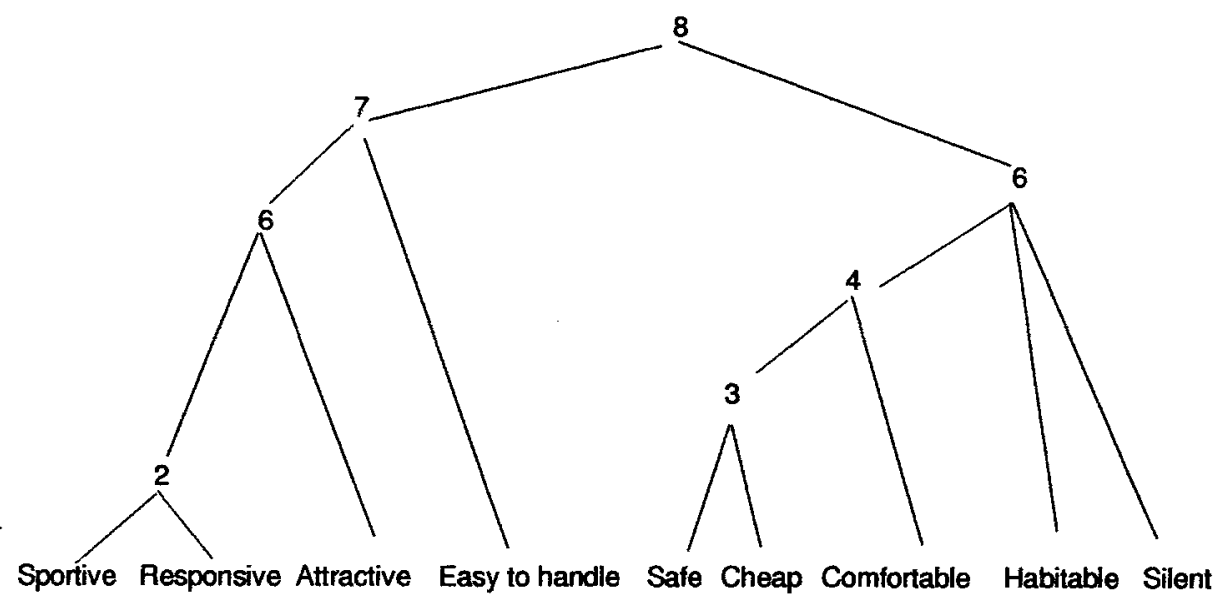

Fig. 2. Characteristic dependency tree obtained from the car rating grid (extracted from [46])

\subsection{Topoi and Acquisition Methods}

Can topoi be naturally extracted, in the framework of some well known acquisition methods? We will focus on the methods KADS and KOD. By lack of room, we shall not give a detailed description of them and we will suppose their principles and their vocabularies known: the reader can find detailed descriptions of such methods in the papers cited as references.

Acquisition of Topoi through KADS Method. Let us briefly recall that KADS $[11,10,42,49]$ advises the knowledge engineer to build a model of the expertise, this model being described with four levels: the levels domain, inference, task and strategy. In order to help the knowledge engineer to build the expertise model corresponding to his application, KADS offers him a library of interpretation models: such models offer predefined descriptions of the levels inference, task and strategy, for different problem classes. Such interpretation models allow the knowledge engineer to interpret the first data elicited from the expert and to acquire the domain level in a top-down way, while being guided by the interpretation model adequate for the considered application.

Exploitation of KADS Domain Layer. The introduction of topoi in the conceptual model is rather a matter for KADS domain level: as this layer includes the domain concepts, their structures and the relations between such concepts, the topoi are particular cases of relations between domain concepts. But what help can KADS offer for the acquisition of topoi since the contents of this level domain is not predefined (this layer domain is not part of the predefined descriptions offered by KADS in its library of interpretation models)? This contents, 
non generic, depends on the application: so, the necessity of topoi acquisition depends on the application. The next sections will indicate how a help is possible, thanks to KADS interpretation models.

Exploitation of Interpretation Models. The resolution of some problem classes seems to need often the use of gradual knowledge (for example, in design problems, as it was noticed by [23,4]). Such a frequent use of topoi should have an influence on the description of the KADS interpretation models corresponding to such problem classes. Topoi must be linked to at least one of the three higher levels (inference, task and strategy) since only such levels are described in the KADS library. So, how to link the topoi to the primitives of description of those three levels?

Exploitation of KADS Inference Layer. The KADS inference layer is described through knowledge sources and roles, notions that seem to us more abstract than topoi.

- Topoi and KADS Knowledge Sources. The knowledge sources cited in [11] allow to modify concepts (assign-value, compute), to generate concepts (instantiate, classify or identify, generalize, abstract, specify or refine), to differentiate concepts (compare, match) and to handle structures (assemble, sort, decompose, transform, parse). But, in KADS, inference methods can be associated to a knowledge source: they indicate how the inferences underlying this knowledge source are carried out at the domain layer. So, topoi may be used in such inference methods: KADS could indicate that, for a given knowledge source (for example, assign-value or compute) appearing in a given inference structure, the associated inference method will consist of the creation of particular topoi or of a propagation of topoi or will verify some topoi considered as constraints.

- Topoi and KADS Roles. The figure 3 shows where topoi could be inserted in the typology of roles presented in [11] page 41.

If topoi were enough abstract to be handled at the inference layer, it would be tempting to add topos or topoi base to the KADS predefined metaclasses. But where would those new metaclasses be inserted in the typology of roles presented in [11] ? A topos or a topoi base may be considered as a role refining the metaclasses system description or constraint, but it could also be seen as an intermediary role of domain knowledge (specializing for example the metaclass system_model) or even as a solution (refining the metaclasses design or model). The fact that a metaclass topos or topoi base may be inserted at different places of the metaclass typology confirms the fact that topoi should not appear as primitives of description of the inference level, but as parts of the domain layer. During the resolution of a given problem, they can then play several different roles: a topoi network can constitute a system description, a topos can be seen as a constraint, a topoi network can be considered as a model of a system (for example, a causal model) and the description of a design problem solution can include a topoi network. 
Metaclass

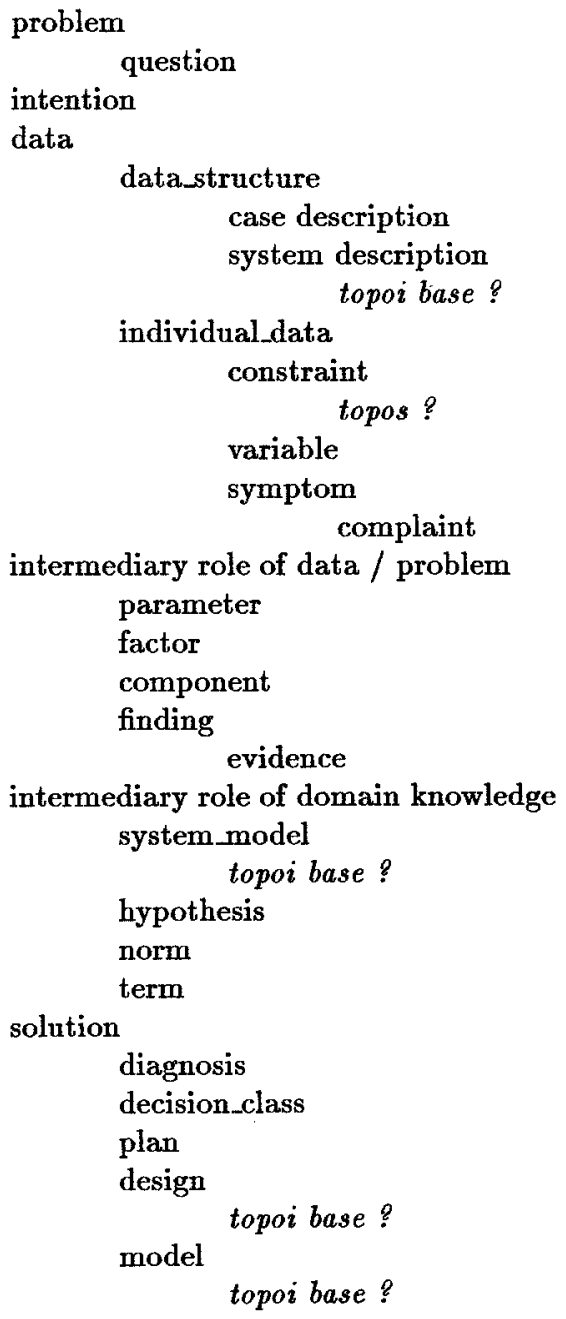

Fig. 3. Topoi in the typology of KADS roles (extracted from [11])

Exploitation of Task and Strategy Layers. The task layer describes structured tasks and their decomposition. The strategy level describes the adaptation of the problem solving plans to the context. None of both levels seems relevant to evoke directly topoi.

Exploitation of KADS Interpretation Model Library. The description of some KADS interpretation models can evoke topoi.:

- Analysis tasks:

- Diagnosis by causal tracing [11] is a systematic diagnostic relying on the possibility to explain the system functioning through causal relations. 
It begins by selecting a model of the system, model that consists of a causal network. So, in the KADS description of this interpretation model, it can be indicated that this causal network can be a topoi network. If the knowledge engineer chooses this interpretation model as the most adequate for his application, he must then try to acquire this topoi network.

- Monitoring verifies whether, during its execution, a system respects a reference model of the system. This model can consist of a topoi base. In [11], a patho-physiological model of human body for a task of intensive care monitoring is given as example of system model. The analysis of the patho-physiological model described in [24] shows that such a model can rely on topoi: indeed, the model proposed by [24] includes topoi even though the term is not explicitly used by the author.

- Prediction allows to determine what will happen to a system in a given situation. Prediction upon a system relies on a description of the system and on a model of the system. This system model can consist of a topoi base. For example, the qualitative model allowing to perform prediction for continuous process control in [39] includes a network of topoi (even though the term was not used by the authors). In [11], the authors distinguish two interpretation models for prediction (behaviour prediction and value prediction) and they stress that values concerned in value prediction are generally quantitative. So, it seems plausible that the use of a topoi base as a model of the system is more useful for behaviour prediction.

\section{- Modification tasks:}

The description of modification tasks (repair, remedial, control, maintenance) in [11] is not enough precise to indicate whether a topoi base may be used as a model of the considered system or as a system description. Indeed, [11] proposes no inference structure or task structure for those interpretation models. However, in [11], the authors notice relations between some analysis tasks and some modification tasks: generally, modification tasks are performed after a diagnosis task identified a malfunction and they aim at restoring the system in its normal functioning. For example, heuristic classification is linked to remedial and to repair, diagnosis by causal tracing is linked to remedial and to repair, diagnosis by localisation is linked to repair and monitoring is linked to control and to maintenance. We suppose that a modification task following an analysis task where topoi were used (diagnosis by causal tracing, monitoring) may also take into account such topoi.

\section{- Synthesis tasks:}

- According to the inference structure proposed in [11], a design task starts with an informal problem statement consisting of (a) an informal specification of the problem, (b) constraints upon the system to be designed or 
(c) requirements. An analysis of this informal statement (through expansion or transformation of this statement) allows to get a formal specification of the system to be designed. This formal specification can consist of design criteria, design constraints, design variables, design goals, a functional specification or a component specification. By selection or aggregation on this formal specification, a conceptual model is built: it can consist of (a) an abstract model, (b) the artefact characteristics or (c) a sketch. From this conceptual model, thanks to a transformation / expansion or to a refinement, the detailed design of the system is obtained: it consists of a full specification of the components and of a full structural description. Such is the general description of the design process proposed in [11]. The authors distinguish hierarchical design, transformational design, incremental design and configuration and give more precise inference structures for some of those design tasks.

Since several concrete experiments confirm the importance of topoi in design applications [23, 4], how to introduce them naturally in the interpretation model proposed by KADS for the general design task? In fact, it is not evident if the topoi base must be part of the informal problem statement (for example, the constraints upon the system to be designed) or if it must rather appear in the formal specification (design constraints, functional specification...) or if it is part of the conceptual model of design. It depends on whether one consitiers the notion of topoi as formal or not. We prefer to see the topoi base appear as a part of the formal specification. It is clear that the topoi base cannot constitute the detailed design model since, as explained in to [11], no additional specification and no later choice need be performed on this detailed design model in order to build the system. And without doubt, some work in order to detail the quantitative aspects underlying topoi is necessary before obtaining the final system from the topoi base.

- For planning and modelling tasks, since [11] proposes no inference structuce or task structure, we don't have enough elements to suggest whether a topoi base can be useful for such tasks. Perhaps, some specific kinds of modelling tasks require the use of topoi but this intuition needs be argumented or at least confirmed by some examples.

Conclusion. In conclusion, this study shows how KADS can be extended in order to indicate explicitly that some roles (system model, system description, formal specification...) appearing in the inference structures offered for some interpretation models, can be played by a topoi base. Thanks to such an extension, KADS can guide the knowledge engineer and advise him to acquire topoi. Such topoi will be part of the domain layer, in the conceptual model of the application, and will be linked to the adequate metaclasses of the inference level.

Remark: This reflection on the jnsertion of topoi within KADS framework is situated at the knowledge level. 
Acquisition of Topoi through KOD. KOD $[47,1,48]$ advises the knowledge engineer to build a "practical model", a "cognitive model" and an "information model". Each of such models is constituted of three kinds of KOD entities, representing respectively the aspects description, action and declaration. Intuitively, the descriptive aspect corresponds to "objects" viewpoint, the action aspect corresponds to the dynamic aspects of knowledge ("methods" or "tasks"), and the declarative aspect corresponds to "rules" or "constraints".

The knowledge engineer starts with the data elicited from the expert and KOD helps him to build the practical model and the cognitive model. This section will study if the notion of topos can be expressed naturally in the KOD entities handled in both models.

Practical Model. Thanks to the analysis of the expert's discourse, the knowledge engineer constructs the practical model: he must build the taxems, actems and schemems corresponding respectively to the aspects description, action and declaration.

Taxems are detected by expressions such as "is-a", "described-by", "is-situated$i n$ " indicating respectively a taxonomic unit, a property, a localisation, etc. We propose to extend KOD by allowing the detection of expressions able to reveal a topic link (for example, "The more .. the more" or "all the more so since", etc.). Above, we gave examples of such expressions. Moreover, an expression such as "The stronger the wave, the thicker the breakwater" can reveal the existence of two taxems wave and breakwater, having respectively the properties strength and thickness.

Actems are verbal manifestations of action units. An actem describes a state change caused by a sender on a receiver, perhaps by using resources and under some constraints. This state change can sometimes correspond to a value modification of the receiver ; this receiver may be linked by a topos to the entity represented by the sender, and the constraint concerning the sender or the action triggering may be the sender value modification. So, a topos "The more $X$ is $A$, the more $Y$ is $B$ " expressed in the expert's discourse can be described by an actem having a sender $X$, a receiver $Y$, this latter being transformed from the state $B i$ to the state $B j$, provided that the value of $X$ was modified from $A i$ to $\mathrm{Aj}$ and that the variation direction from $\mathrm{Bi}$ to $\mathrm{Bj}$ is the same as from $\mathrm{Ai}$ to $\mathrm{Aj}$.

The declarative aspect of knowledge appears in the practical model through constraints (called modal schemems) or through inferences (called causal schemems). The topoi detected in the expert's discourse can appear as constraints or as inferences: for example, the topos "The more $X$ is $A$, the more $Y$ is $B$ " may be considered as a constraint or may be described as the inference "If $X$ is $A$, then $Y$ is $B$ ". However, the description of the topos through an inference rule suffers from a loss of information on the gradual aspects of the link between $X$ and $\mathrm{Y}$.

Cognitive Model. The cognitive model allows a structuration of the practical model. 
A taxonomy is a hierarchical representation of the physical world, as handled by the expert. Insofar as, in the taxonomies of the cognitive model, only the specialization link is useful, it is difficult to handle explicitly topoi in taxinomies since, in KOD, relations between entities remain implicit behind the attributes of the taxonomic units.

The dynamic aspect of knowledge appears through sequences of actions, called actinomies. Since, as shown above, an actem can sometimes express a topos, an actinomy can express a chain of successive topoi. For example, the succession of topoi "The more $X$ is $A$, the more $Y$ is $B$ " and "The more $Y$ is $B$, the more $Z$ is $C^{n}$ can be expressed by an actinomy made of two action units, allowing to describe the successive transformations of $Y$ and of $Z$, due to a modification of $\mathrm{X}$.

The constraints (resp. inferences) appearing in the practical model are the verbal manifestations of modal (resp. causal) abstractions, called behavioural schemata (resp. interpretation schemata). So, the declaration aspect of knowledge appears in the cognitive model through such schemata: through them, the knowledge engineer tries to abstract the declarative aspects he modelled in the practical model. Topoi can be described naturally as behavioural schemata: for example, a topos can be the behavioural schema underlying several constraints verbally expressed by the expert. A topos can also be an interpretation schema, abstracting several inferences verbally expressed by the expert.

Conclusion. The declaration viewpoint is the aspect where the topoi are the most natural to express in KOD, both in the practical model and in the cognitive model. It is not surprising since topoi can be seen either as constraints or as inference rules.

Topoi and Generic Tasks. As described in $[13,15]$, a generic task is characterized by the knowledge it uses, the organization of this knowledge, and the inference strategies associated to this generic task. Each generic task corresponds to an adapted knowledge acquisition strategy. For example, in [13], the authors describe hierarchical classification and design by selection and plan refinement: they detail the implications of hierarchical classification for knowledge acquisition.

So, for some generic tasks, topoi could be part of the knowledge to organize, because, for such tasks, they would be a type of knowledge often handled by the experts solving this class of problems. We did not try to integrate topoi in the generic tasks already proposed by Chandrasekaran \& al. But we would suggest to introduce a new generic task, called simulation of a system by topoi propagation: topoi would be part of the knowledge; the components of the system to be designed or the entities influencing its design would be organized through topoi networks. The inference strategy associated to this new generic task would correspond to a propagation of modifications along the topic links (as, for example, the propagation proposed in 3DKAT [25]). A specific knowledge acquisition strategy, for example inspired of 3DKAT strategy, would be associated to this new generic task. 
Topoi and Problem Classes. The study of different problem classes is important in research on knowledge acquisition, as proved by Clancey's work on a typology of problem classes [16], or KADS interpretation model library [11] or the generic task approach [15] or research on problem solving methods [36, 32]. Topoi seem to be often used in some problem classes: our analysis of the different problem classes for which KADS offers an interpretation model confirms the usefulness of topoi for diagnosis by causal tracing, for monitoring, for behaviour prediction and for design. Moreover, several experiments presented by researchers attest that experts use topoi in numerous applications. For example, in diagnosis, examples of topoi for diagnosis of highways are given in [27]. As noticed above, even though the term "topos" is not used by the authors, the patho-physiological model described in [24] in the framework of a system for aid to medical diagnosis comprises topoi and the simulation performed on this deep model (which is a qualitative model) is an example of reasoning possible for exploiting topoi. It is not surprising, as the classes of problems processed by qualitative physics seem to be classes of problems where topoi should be used naturally. Likewise, the prediction and diagnosis for the system of aid to continuous process control described in [39] rely on topoi (even though the authors don't use the term "topos").The problems of simulation (in particular, qualitative simulation) seem also to benefit from making topoi explicit. In design, examples are presented in [23] (geostationary satellite configuration) and in [4] (design of aerospace products). An experience of "reverse engineering" on an already existing expert system for breakwater design [37] allowed to extract a posteriori topoi for this routine configuration application.

All those examples cited in the literature attest the variety of the problem classes for which experts use topoi: such problem classes would benefit from a method or a tool helping to topoi acquisition.

\subsection{Validation of Acquired Topoi}

The acquired topoi must be validated by the expert. If the topoi have not been expressed directly by the expert, but obtained thanks to an indirect elicitation technique such as rating grids, or thanks to the exploitation of a method such as KADS, as described above, the expert must agree with such topoi. But even if the expert expresses by himself some topoi (as shown in the examples cited in the section 3.1.1), a verification in comparison with other topoi or with the "quantitative knowledge" elicited from the expert must always be carried out.

Verification of a Topos Relatively to Other Topoi. The knowledge engineer must gather all the topoi linking two given entities (for example, two parameters of the system to be designed or diagnosed, such as the age of the roadway and the solidity of the foundation). If two topoi are contradictory (e.g. one corresponds to "The older the roadway, the worse the foundation" and the other expresses that "The older the roadway, the better the foundation", the expert must confirm whether he maintains both of them. If it is not a mistake, it 
means that some additional information on the validity domain of the topoi is missing: for example, the latter topos may be true only unless the foundation age exceeds a given threshold or only during a given step of the expert's reasoning. So, the knowledge engineer must ask the expert additional conditions of validity, in order to suppress the contradiction [18].

Verification of a Topos Relatively to Quantitative Knowledge. If the expert also gives quantitative information such as an equation about two entities linked by a topos, the compatibility between the quantitative knowledge and the qualitative one must be verified. For example, if the topos "The higher $X$, the higher $Y$ " between two numerical parameters $\mathrm{X}$ and $\mathrm{Y}$ is elicited and if the function allowing to calculate $\mathrm{Y}$ in terms of $\mathrm{X}$ is elicited (either expressed directly by the expert or indirectly deduced from other information), the variation direction of this function must be compatible with the topos (i.e. the function must be increasing). If the function is not monotonic, it means that the topos should be conditional, in order to restrict its validity domain.

Remark: Several ideas proposed in [20] for verifying the consistency of a topoi base can be adapted to the validation of acquired topoi: in particular, the implicit topoi that could be deduced from a chain of elicited topoi must also be validated.

\section{Representation and Implementation of Topoi}

\subsection{Representation of Topoi}

At the symbol level, several knowledge representation formalisms allow to represent topoi: simple production rules, fuzzy production rules [9], object-oriented representation formalisms [23, 25], constraints or dependency relations [29], conceptual graphs [44], knowledge graphs [3,31,35]. For lack of room, the use of the different formalisms will not be detailed in this paper.

\subsection{Topoi and Qualitative Physics}

Topoi can be considered as a particular case of qualitative knowledge and can be formalized through the main formalisms of qualitative physics. But such a formalization must be validated by the expert, taking into account the semantics of such formalisms.

In de Kleer's component based approach [19], the positive topoi "The higher $x$, the higher $y$ " and "The lower $x$, the lower $y$ " can be formalized by the confluence $d x=d y$ and the negative topoi "The higher $x$, the lower $y$ " and "The lower $x$, the higher $y$ " by the confluence $d x=-d y$. As noticed in [14], there is a loss of information, as de Kleer's confluences are not oriented, contrarily to topoi.

In Forbus's process qualitative theory [28], the positive topoi can be formalized by the qualitative proportionality relation $y \alpha_{Q+} x$ and the negative topoi by $y \alpha_{Q-x}$. 
In Kuiper's qualitative constraints approach [34], the positive topoi are expressed through the functional constraint $y=M^{+}(x)$ and the negative topoi through $y=M^{-}(x)$.

Topoi allow to establish a link between qualitative physics and the common sense evoked by Hayes [30]. Notice that, according to the case, the expert can express: (a) the quantitative equations linking the variables, (b) the qualitative equations used in qualitative physics, (c) the topoi corresponding to the qualitative links between the variables.

Remark: In [14], the link between qualitative physics and topoi is studied thoroughly, in a purpose of explanation generation: the authors indicate how to extract topoi from the qualitative information described through qualitative physics formalisms, while our work aims at formalizing and representing the topoi elicited from the expert, through qualitative physics formalisms, at the symbol level.

\section{Conclusions}

As attested by the research cited in the introduction, topoi are useful at various phases of a KBS life cycle: help to design of a knowledge-based system [18], reasoning on a topoi base [23, 25, 22], explanations based on topoi [41, 20,21], validation based on topoi [18].

Detailing all topoi uses goes beyond the scope of this paper that focuses on knowledge acquisition. The main ideas presented in this article were:

- the study of topoi at Newell's knowledge and symbol levels,

- the study of topoi elicitation, using some knowledge elicitation techniques such as rating grids,

- the study of topoi acquisition using the methods KADS and KOD, and the generic task approach. This study allowed us to emphasize the link between topoi and problem classes.

- the evocation of different possible representations of topoi at the symbol level, and topoi formalization through qualitative physics formalisms.

Such ideas are different from previous research: in [23, 25, 22], a knowledge acquisition tool based on topoi was proposed, and in [9], the acquisition of gradual rules based on fuzzy implication was studied. In this article, we analysed a knowledge elicitation technique (rating grids) and several knowledge acquisition methods (KADS, KOD) in order to help the knowledge engineer to acquire topoi, and such an approach seems to be original.

The ideas presented in this article can suggest several research directions: a thorough study of the different problems raised by the analysis of topoi at the knowledge level, a rigorous study of the semantics of topoi, an implementation of topoi using the different representations evoked above and a comparison between their respective advantages and drawbacks, and, last, a study of the link between topoi acquisition and their exploitation in the final knowledge-based system. 
Of course, topoi don't allow to represent everything. Among their limits, in engineering problems, topoi are not sufficient as they are too few informative without a minimum of information on the orders of magnitude of the handled variables. So, we stress the interest of research for adding information on order of magnitude to qualitative knowledge such as topoi.

Our approach can be generalized: if a specific type of knowledge is recognized as important in some classes of problems, then some knowledge elicitation techniques and some knowledge acquisition methods can be analysed in order to see if they help to elicit naturally that kind of knowledge. Concerning the method KADS, it is interesting to study which primitives of the inference layer can be linked to this kind of knowledge. The link between this type of knowledge and the problem classes where such knowledge is useful indicates in which interpretation models KADS can suggest to acquire this type of knowledge. In the framework of KOD method, the different KOD entities appearing in the KOD models must be analysed in order to see which of them allow to express this type of knowledge. For generic task approach, either this type of knowledge can be linked to already existing generic tasks (for example, it may be part of the knowledge to organize) or, in some cases, it may be worthy to create an adequate generic task handling this type of knowledge, exploiting it with a convenient inference strategy and offering a specific strategy to acquire this type of knowledge.

\section{References}

1. P. Albert and C. Vogel. Kod-station un environnement intégré pour le génie cognitif. Génie logiciel et systèmes experts, (19):28-30, Juin 1989.

2. J. C. Anscombre and O. Ducrot. L'argumentation dans la langue. Mardaga, Bruxelles, 1983.

3. R. D. Bakker. Knowledge graphs: representation and structuring of scientific knowledge. PhD thesis, University of Twente, 1987.

4. N. Bonnardel. Le rôle de l'évaluation dans les activités de conception, Juillet 1992. Thèse de Doctorat en Psychologie, Université de Provence.

5. J. H. Boose. Personal Construct Theory and the Transfer of Human Expertise. In Proc. of AAAI-84, pp. 27-33, Austin, Texas, USA, August 1984.

6. J. H. Boose. A Knowledge Acquisition Program for Expert Systems based on Personal Construct Psychology. Int. Journal of Man-Machine Studies, 23:495525, 1985.

7. J. H. Boose and J. M. Bradshaw. Expertise transfer and complex problems: using AQUINAS as a knowledge-acquisition workbench for knowledge-based systems. International Journal of Man-Machine Studies, 26:3-28, 1987.

8. J. H. Boose, J. M. Bradshaw, C. M. Kitto, and D. B. Schema. From ETS to AQUINAS: Six years of Knowledge Acquisition Tool Development. In Proc. of $K A W^{\prime} 89$, pp. 5.1-5.17, Banff, Canada, October 1989.

9. B. Bouchon-Meunier and S. Despres. Acquisition numérique/symbolique de connaissances graduelles. In Actes des 3èmes Journées nationales du PRC-GDR Intelligence Artificielle, pp. 127-138, Paris, La Défense, Mars 1990. Hermès.

10. J. Breuker and B. Wielinga. Models of Expertise in Knowledge Acquisition. In Guida and Tasso, editors, Topics in Expert System Design. North-Holland, Elsevier 
Science Publisher B.V., 1989.

11. J. Breuker, B. Wielinga, M. van Someren, R. de Hoog, G. Schreiber, P. de Greef, B. Bredeweg, J. Wielemaker, and J.-P. Billault. Model Driven Knowledge Acquisition: Interpretation Models, 1987. Del. A1, Esprit Proj. 1098, Memo 87.

12. S. Bruxelles and P. Y. Raccah. Information and argumentation; the expression of the consequence. In COGNITIVA'87, vol. 1, pp. 445-452, Paris, May 1987.

13. T. Bylander and B. Chandrasekaran. Generic Tasks in Knowledge-Based Reasoning : the 'Right' Level of Abstraction for Knowledge Acquisition. International Journal of Man-Machine Studies, 26:231-244, 1987.

14. F. Cerbah, C. Fournier, and P.-Y. Raccah. Qualitative Reasoning and Argumentation: a Study of some Affinities when Generating Causal Explanations. In M. G. Singh and L. Travé-Massuyès, eds, Decision Support Systems and Qualitative Reasoning, pp. 229-234, North-Holland.

15. B. Chandrasekaran. Towards a Functional Architecture for Intelligence Based on Generic Information Processing Tasks. In Proc. of the 10th IJCAI, volume 2, pp. 1183-1192, Milan, Italy, August 1987.

16. W. J. Clancey. Heuristic classification. Art. Int., 27(3):289 350, Dec. 1985.

17. W. J. Clancey. The knowledge level reinterpreted: Modeling how systems interact. Machine Learning, 4:285-291, 1989.

18. H. Davis. Using models of dynamic behavior in expert systems. In 9th Int. Workshop Expert Syst. 6 their Appl., pp. 393-404, Avignon, France, May-June 1989.

19. J. de Kleer and J. S. Brown. A qualitative physics based on confluences. Artificial Intelligence, 24:7-83, 1984.

20. R. Dieng. Generation of topoi in expert systems. Communication, Cognition and Artificial Intelligence, 6(4):329-340, 1989.

21. R. Dieng. Knowledge-based, relation-based and learning-based explanations. In Proceedings of the 5th Workshop on Explanations, Manchester, UK, April 1990.

22. R. Dieng. Models of Problem Solving for Knowledge Acquisition: Modelling Sisyphus'91 Office Assignment Problem with 3DKAT. In M. Linster, editor, SISYPHUS'91: Models of Problem Solving, GMD Report n. 663, July 1992.

23. R. Dieng and B. Trousse. 3DKAT, a Dependency-Driven Dynamic-Knowledge Acquisition Tool. In Proceedings of the 3rd International Symposium on Knowledge Engineering, pp. 85-93, Madrid, Spain, 17-21 October 1988.

24. P. Dugerdil. Deep model reasoning in medical expert systems. In 10th Int. Workshop Expert Systems 6 their Applications: 2nd Generation Expert Systems, pp. 85-96, Avignon, May-June 1990.

25. M.-P. Epp and N. Riera. 3DKAT: Un outil d'aide à l'acquisition des connaissances, September 1989. Rapport de stage, DESS-ISI \& INRIA-Sophia-Antipolis.

26. E. Faisandier. Introduction des conditions dans l'outil d'acquisition des connaissances $3 D K A T$, Nov. 1989. Rapp. de stage, ENSIMAG \& INRIA-Sophia-Antipolis.

27. C. Ferraris. Acquisition des connaissances et raisonnement dans un univers multiagents: application à la prise de décision en génie civil urbain, Février 1992. Thèse de Doctorat en Informatique, Université de Nancy I.

28. K. D. Forbus. Qualitative process theory. Artificial Intelligence, 24:85-168, 1984.

29. M. Fornarino and A.-M. Pinna. Un modèle objet logique et relationnel: le langage OTHELO, Avril 1990. Thèse de Doctorat en Informatique, Université de Nice.

30. P. Hayes. The second naive physics manifesto. In Hobbs and Moore, eds, Formal Theories of the Common-Sense World, pp. 1-35. Ables, Norwood, NJ, 1985.

31. P. James. Structuring Knowledge using Knowledge Graphs. In Proc. of $E K A W^{\prime} 91$, Crieff, Scotland, United-Kingdom, May 1991. 
32. W. Karbach, M. Linster, and A. Voss. Models, methods, roles and tasks: many labels - one idea? Knowledge Acquisition, 2:279-299, 1990.

33. G. A. Kelly. The Psychology of Personal Constructs. Norton, 1955.

34. B. J. Kuipers. Qualitative simulation. Artificial Intelligence, 29:289-338, 1986.

35. S. Lapalut. Un modèle d'acquisition des connaissances qualitatives en conception pour KATEMES. Rapp. de DEA, Univ, de Caen \& INRIA, Sept. 1992.

36. J. McDermott. Preliminary Steps Toward a Taxonomy of Problem-Solving Methods. In Sandra Marcus, editor, Automating Knowledge Acquisition for Expert Systems, pp. 225-266. Kluwer Academic Publisher, 1988.

37. B. Neveu. EXPORT: an expert system in breakwater design. In ORIA'87, Artificial Intelligence and sea, pp. 11-17, 1987.

38. A. Newell. The knowledge level. Artificial Intelligence, 18:87-127, 1982.

39. J. M. Penalva and Y. Sicard. DIAPASON: prédiction et diagnostic pour la conduite de procédés continus. In ERGO-IA'90, pp. 196-207, Biarritz, Sept. 1990.

40. H. Prade. Raisonner avec des règles d'inférence graduelles: une approche basée sur les ensembles flous. Revue d'Intelligence Artificielle, 2:29-44, 1988.

41. P. Y. Raccah. Modelling argumentation and modelling with argumentation. Argumentation, 4, 1989.

42. G. Schreiber, B. Wielinga, and J. Breuker. The KADS Framework for Modelling Expertise. In Proc. of EKAW'91, Crieff, Scotland, UK, May 1991.

43. M. L. G. Shaw. A Grid-Based Tool For Knowledge Acquisition: Validation with Multiple Experts. SIGART Newsletter, Knowledge Acquisition Special Issue, 108:168-169, April 1989.

44. J. F. Sowa. Conceptual Structures: Information Processing in Mind and Machine. Reading, Addison-Wesley, 1984.

45. L. Steels. Knowledge Systems. 1991. Draft edition, VUB AI Lab, Brussels, Belgium.

46. L. Vandenbreghe. Techniques de recueil d'expertise. In Séminaire Méthodologies d'acquisition de la connaissance, IIIA, Compiègne, Sept. 1991.

47. C. Vogel. Génie Cognitif. Masson, 1988.

48. C. Vogel. How to Qualify Knowledge-Based Systems. In Proceedings of the 4th COMPASS Conference, 1989.

49. B. J. Wielinga, A. T. Schreiber, and J. A. Breuker. KADS: a modelling approach to knowledge engineering. Knowledge Acquisition, 4:5-53, 1992. 